\title{
Regulation of Cholesterol Metabolism in Adrenal Cortex: Effects of Apoproteins on Cholesterol Esterase in Rat Adrenal Glands
}

\author{
Tetsuo NiShiKAWA, Keiji MIKAMI*, Akiko YOSHIDA**, \\ Masao OMURA, Yasushi TAMURA** and Yasushi SAITO** \\ Department of Medicine, Yokohama Rosai Hospital, Kanagawa 222, \\ *Department of Medicine, Chiba Municipal Hospital, Chiba 260, \\ **Second Department of Internal Medicine, School of Medicine, \\ Chiba University, Chiba 260, Japan
}

\begin{abstract}
We have investigated the effects of apoproteins on cholesterol esterase (CEase) in rat adrenal glands in order to clarify the mechanism of synthesis of free cholesterol which is the most important substrate for steroidogenesis. We prepared lipid mixtures containing cholesteryl oleate plus apoproteins with and without phosphatidylcholine as a substrate for CEase in order to investigate the effect of the substrate state on CEase. The substrate containing only cholesteryl oleate and apo-HDL increased both acid and alkaline CEase activities. Both acid and alkaline CEase activities were also increased by a substrate containing apo-HDL plus cholesteryl oleate and phosphatidylcholine more than by a substrate containing cholesteryl oleate plus apo-LDL with phosphatidylcholine or cholesteryl oleate with phosphatidylcholine. We have already reported that phosphatidylcholine is an important factor for the regulation of adrenal CEase. Therefore, the present studies show that apoproteins as well as phosphatidylcholine may be important factors for the regulation of adrenal CEase.
\end{abstract}

Key words: Rat adrenal, Cholesterol esterase, Apoproteins.

(Endocrine Journal 40: 22 1-225, 1993)

PREVIOUS studies in rats $[1,2]$ as well as in man [3-5] have demonstrated that the adrenal gland derives a major portion of its cholesterol from plasma. In contrast to mouse adrenal tumor cells $[6,7]$, guinea pig adrenals [8], and human fetal adrenal glands [9], not only low density lipoprotein (LDL), but also high density lipoprotein (HDL) can be an important source of cholesterol for steroidogenesis in rat adrenal glands [10-12]. We have demonstrated that free cholesterol for steroidogenesis may be supplied by two different organelles (lysosome and microsome), and that the composition of the substrate complex, for example the ratio of esterified cholesterol to phospholipids,

Received: December 1, 1992

Accepted February 19, 1993

Correspondence to: Dr. Tetsuo NISHIKAWA, Department of Medicine, Yokohama Rosai Hospital, 3211 Kozukue-cho, Kohoku-ku, Yokohama City, Kanagawa 222, Japan may play a crucial role in the regulation of adrenal cholesterol esterase (CEase; EC 3.1.1.13) [13]. It has been reported that rabbit lipoproteins cause inhibition of CEase activity in the rabbit aortae [14] and that lipoprotein serves as a good substrate for hydrolysis of esterified cholesterol in dog plasma [15]. Moreover, the evidence indicating that apoHDL plays a role in the cholesterol uptake process in the rat adrenal glands [16] indicates that not only lipoproteins but also apoproteins may play an important role in the regulation of adrenal steroidogenesis. It has recently been reported that there is strong apoE synthesis in the adrenal gland, suggesting that apoE may play a role in regulating the utilization of cholesterol for steroid production [17]. These experiments were therefore carried out to clarify the effects of apoproteins on adrenal CEase. 


\section{Materials and Methods}

Cholesteryl oleate was purchased from Sigma Chemical Co. Phosphatidylcholine was a gift from Nippon Shoji Co., Osaka, Japan. Other chemicals and solvents were obtained from Wako Chemical Co., Tokyo, Japan and were of analytical reagent grade. Cholesteryl $\left[1-{ }^{14} \mathrm{C}\right]$ oleate $(\mathrm{SA} 51.0 \mathrm{mCi} /$ mmol) was purchased from New England Nuclear Co., U.S.A. The purity of the radioactive cholesteryl oleate was confirmed by thin-layer chromatography.

\section{Animals}

Male Wistar rats weighing from 200 to $250 \mathrm{~g}$ were obtained from Takasugi Animal Co., Urawa, Japan and were maintained on a standard rat pellet diet (Oriental Yeast Co., Tokyo, Japan) and given water ad libitum. They were kept in the animal room prior to sacrifice to minimize stress induced by handling, and were killed by decapitation. The adrenal glands were quickly removed, trimmed free of adherent fat, and homogenized in an ice-cold buffer of $0.25 \mathrm{M}$ sucrose containing 5 $\mathrm{mM}$ Tris- $\mathrm{HCl}$ at a final $\mathrm{pH}$ of 7.4 in Hiscotron (Nichion Co., Funabashi, Japan). The homogenates were centrifuged at $800 \mathrm{~g}$ for $10 \mathrm{~min}$ at $4^{\circ} \mathrm{C}$. The floating lipid layer was carefully removed and the postnuclear supernatant was used as the enzyme solution to estimate CEase activity. The protein concentration was determined by the method of Lowry et al. [18] using bovine serum albumin as standard.

\section{Preparation of lipoproteins and apoproteins}

Lipoproteins were separated from the sera of Wistar male rats (250-300 g of body weight) by the method of Koga et al. [19]. The animals fasted for $16 \mathrm{~h}$ before bleeding. Density criteria for the lipoprotein classes were as follows; $d=1.019-1.040$ for low density lipoprotein (LDL), and $\mathrm{d}=1.063-1.21$ for high density lipoprotein (HDL). The isolated fractions were dialyzed just before using for these experiments for at least $36 \mathrm{~h}$ with three changes of the $10 \mathrm{mM}$ Tris- $\mathrm{HCl}$ buffer at a final $\mathrm{pH}$ of 7.4. The homogeneity of lipoprotein fractions was confirmed by agarose gel electrophoresis and purified lipoproteins were used within 3 weeks of preparation. Concentrations of cholesterol and phospholipids in each lipoprotein were determined as previously reported [20]. Concentrations of total and esterified cholesterol were $154 \mathrm{mg} / \mathrm{d} l$ and $145 \mathrm{mg} / \mathrm{d} l$ in $\mathrm{HDL}$, and 35 $\mathrm{mg} / \mathrm{d} l$ and $31 \mathrm{mg} / \mathrm{d} l$ in LDL, respectively. Concentrations of phospholipids in HDL and LDL were $280 \mathrm{mg} / \mathrm{d} l$ and $34 \mathrm{mg} / \mathrm{d} l$, respectively. Each lipoprotein was delipidated according to the method of Scanu [21]. HDL was delipidated with ethanol-ether $(3: 2, \mathrm{v}: \mathrm{v})$ at $-10^{\circ} \mathrm{C}$, and $\mathrm{LDL}$ was delipidated with ethanol-ether $(3: 1, \mathrm{v}: \mathrm{v})$ at $-10^{\circ} \mathrm{C}$. These delipidated products, all containing less than $1 \%$ lipid, were stored at $-20^{\circ} \mathrm{C}$.

\section{Estimation of CEase activity}

Substrate was prepared and CEase activity was estimated as previously reported $[13,22]$. Cholesteryl $\left[1-{ }^{14} \mathrm{C}\right]$ oleate with unlabeled cholesteryl oleate $(1.6 \mu \mathrm{Ci} / 1 \mu$ mole $)$ was resuspended in $1 \mathrm{ml}$ of $0.2 \mathrm{M} \mathrm{NaCl}$ with and without various concentrations of phosphatidylcholine and apoproteins. These lipid mixtures were sonicated three times within a temperature range of $30-40^{\circ} \mathrm{C}$ for $30 \mathrm{sec}$ in a sonifier. The sonicated lipid mixtures were used as a substrate for estimating CEase activity. The reaction mixture consisted of $50 \mathrm{mM}$ acetate buffer $(\mathrm{pH} 4.5)$ or Tris- $\mathrm{HCl}$ buffer ( $\mathrm{pH} 8.25$ ), $50 \mu l$ of a substrate mixture (final concentration of cholesteryl oleate: $250 \mu \mathrm{M}$ ) and $50 \mu \mathrm{l}$ of enzyme solution, forming a final volume of $200 \mu l$. Acetate buffer was used for determination of acid CEase activity and Tris- $\mathrm{HCl}$ buffer was used for the estimation of alkaline CEase activity. Incubations were performed at $37^{\circ} \mathrm{C}$ for $90 \mathrm{~min}$ and the reaction was stopped by adding $2 \mathrm{~m} l$ of benzene-chloroform-methanol (1.0:0.5:1.2) and $40 \mu l$ of $1 \mathrm{~N} \mathrm{NaOH}$. The mixture was shaken vigorously and then centrifuged at $1000 \mathrm{~g}$ for $20 \mathrm{~min}$. A sample of $0.5 \mathrm{ml}$ of the upper phase was mixed with Triton-toluene scintillation fluid and its radioactivity was determined with a liquid scintillation spectrometer. Under these conditions, $96 \%$ of a sample of $\left[1-{ }^{14} \mathrm{C}\right]$-oleic acid was removed in the upper phase. Blanks incubated in the absence of the tissue fraction were assayed and less than $1 \%$ of total radioactivity present in the incubation was found in the upper phase. CEase activity was calculated by subtracting the disintegrations per min obtained in the upper phase of blanks from the disintegrations per min obtained 
in the oleic acid fraction of usual incubations.

\section{Results}

Effects of the lipid mixtures consisting of only cholesteryl oleate and apoproteins without phosphatidylcholine on acid and alkaline CEase activities are shown in Fig. 1a, and Fig. 1b, respectively. When the substrate contained apo-HDL, acid CEase activity was increased (Fig. 1a). The substrate containing cholesteryl oleate plus apo-LDL produced a decrease in acid CEase activity (Fig. 1a). Alkaline CEase activity was markedly enhanced when the concentration of apo-HDL in the substrate mixtures was increased (Fig. 1b). The substrate mixture containing apo-LDL showed a marked decrease in alkaline CEase activity.

Figs. 2a and $b$ show the effect of the substrate mixtures containing cholesteryl oleate, phosphatidylcholine, and apoproteins on acid and alkaline CEase activities, respectively. Both acid and alkaline CEase activities were much more enhanced by using substrate mixtures containing apo-HDL than by using those containing apo-LDL or no protein, when the concentration of phosphatidylcholine was increased (Figs. 2a and b). Both acid and alkaline CEase activities increased by phosphatidylcholine were synergistically enhanced when simultaneously incubated with apo-HDL. The substrate mixtures containing cholesteryl oleate plus apo-LDL with phosphatidylcholine showed almost the same enhancement of both acid and alkaline CEase activities as those containing only cholesteryl oleate and phosphatidylcholine.

\section{Discussion}

It has been demonstrated that adrenocortical lipid droplets contain cholesterol esters, phospholipids and protein [23]. We have already reported that both acid and alkaline CEase activities were markedly enhanced when the concentration of phosphatidylcholine in the lipid mixture used as substrate was increased [13]. In order to investigate the effect of the substrate state on acid and alkaline CEase, we prepared lipid mixtures containing cholesteryl oleate plus apoproteins with and without phosphatidylcholine. The substrate containing only cholesteryl oleate and apo-HDL increased both acid and alkaline CEase activities. This suggests that apo-HDL may be one of the factors involved in enhancing CEase activity as well as phosphatidylcholine. The present data also demonstrated that the substrate containing cholesteryl oleate plus apo-LDL with and without phosphatidylcholine did not increase the CEase activities, suggesting that such a substrate may not be suitable for activating CEase.

It has been reported that the protein and cholesterol ester components of the internalized LDL are hydrolyzed within the lysosome of a mouse adrenal tumor cell [6, 7]. On the other hand, it has been reported that utilization of HDL cholesterol does not require endocytosis and lysosomal degradation of the entire HDL particle in the rat adrenal [11]. We have already reported that CEase is located in both the lysosomal and microsomal fractions of the rat adrenal [13]. It is therefore speculated that internalized HDL may be degradated by lysosomes in the rat adrenal. However, there have not yet been any reports of the effect of the protein components of the internalized lipoproteins on various adrenal functions. Quite interesting are the results of the present studies showing a remarkable enhancement of acid and alkaline CEase activities when the substrate mixture contained cholesteryl oleate plus apo-HDL with and without phosphatidylcholine. In previous investigations, in rat HDL there was a large amount of two apoproteins (apoA and apoE) $[24,25]$, and 50 to $60 \%$ of circulating cholesterol in the rat was found in HDL [24]. It is also reported that HDL can be an important source of cholesterol for steroidogenesis in the rat adrenal [10-12]. The present study clearly indicates that apo-HDL as part of the substrate for CEase may play a crucial role in the regulation of CEase in rat adrenals, although further experiments will be needed in order to clarify the exact effect of each apoprotein, including apoA and apoE, on adrenal CEase.

In conclusion, the present study has demonstrated that the apoproteins which are components of lipoproteins may regulate adrenal CEase. ApoHDL rather than apo-LDL seems to be one of factors involved in the activation of rat adrenal CEase as well as phosphatidylcholine. 


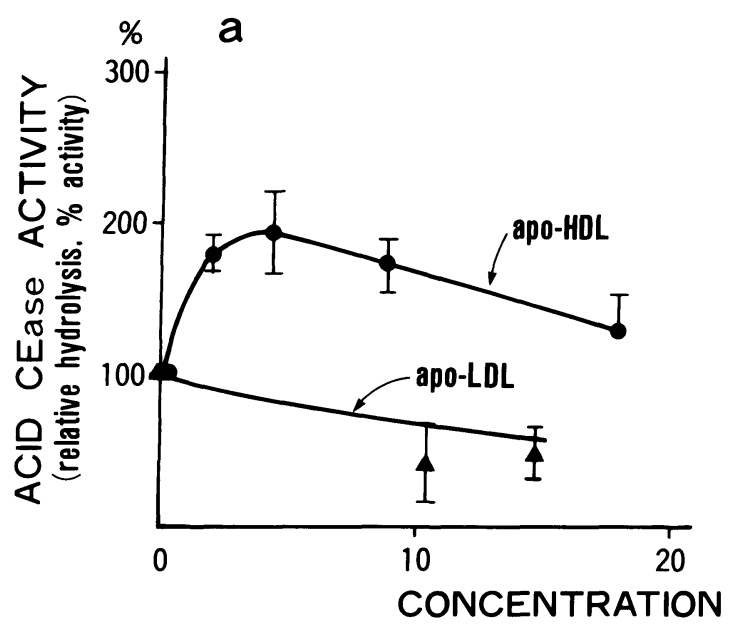


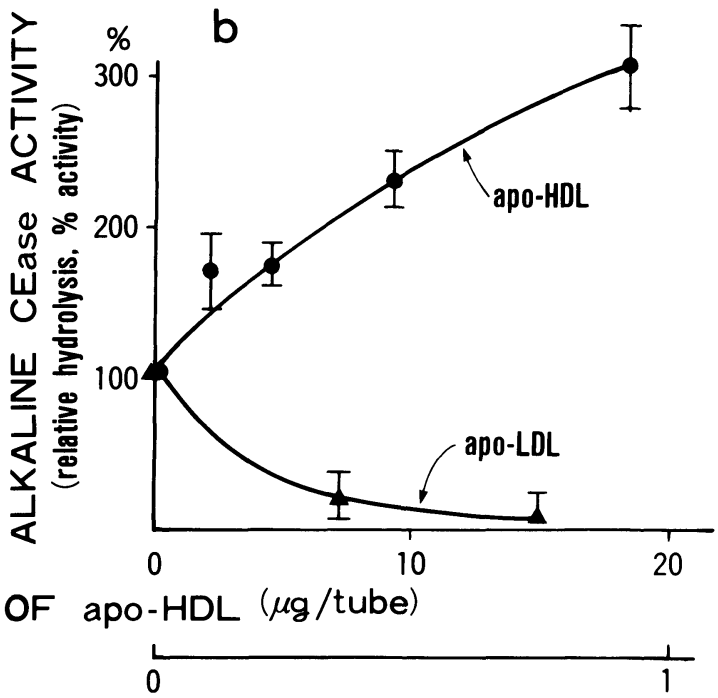

CONCENTRATION OF apo-LDL $(\mu \mathrm{g} /$ tube)

Fig. 1. Effects of the substrate mixtures containing only cholesteryl oleate and apoproteins on CEase activity. The left panel (Fig. 1a) and the right panel (Fig. 1b) show the effects of the substrate mixtures on the acid and alkaline CEase activities, respectively. The substrates were prepared by sonicating a mixture which contained $250 \mu \mathrm{M}$ of cholesteryl $\left[1{ }^{14} \mathrm{C}\right]$ oleate $(0.08 \mu \mathrm{Ci} /$ assay $)$ and various amounts of apoproteins. Fifty $\mu l$ of the sonicated mixtures were obtained and added to the incubation mixtures. Postnuclear supernatant (100 $\mu \mathrm{g}$ of protein per assay) was used. Results are expressed as the mean \pm SEM. ( $n=4$ different experiments).

a

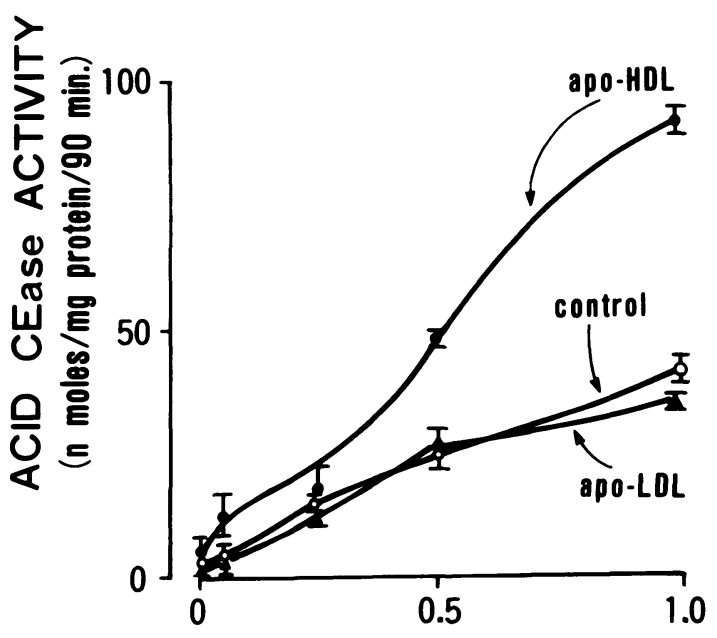

b

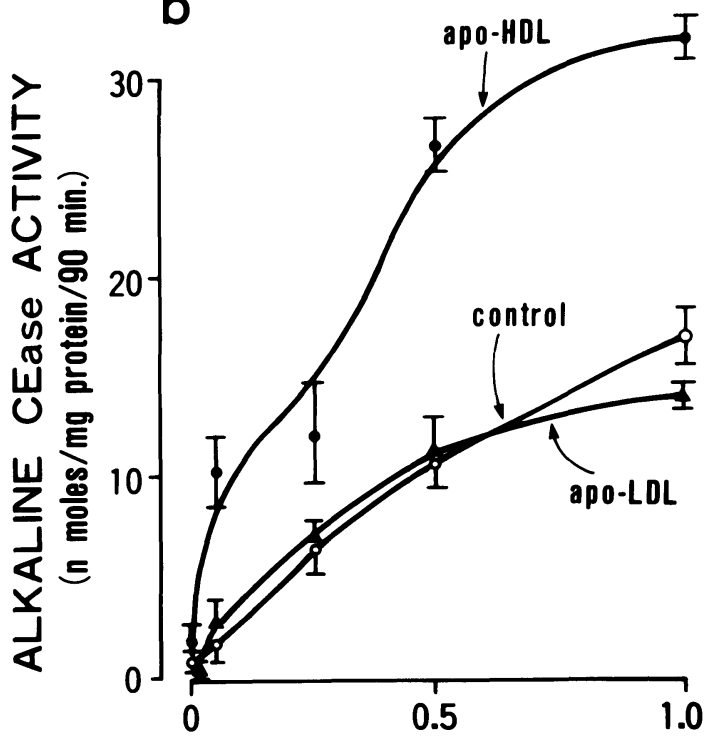

\section{CONCENTRATION OF PHOSPHATIDYLCHOLINE (mM)}

Fig. 2. Effects of the lipid mixtures containing cholesteryl oleate, phosphatidylcholine, and apoproteins on CEase activities. The left panel and the right panel show the effects of the lipid mixtures used as a substrate on acid (Fig. 2a) and alkaline CEase (Fig. 2b), respectively. The substrates were prepared by sonicating a mixture which contained $250 \mu \mathrm{M}$ of cholesteryl $\left[1-{ }^{14} \mathrm{C}\right]$ oleate $(0.078 \mu \mathrm{Ci} /$ assay $)$, various amounts of phosphatidylcholine, and each lipid-depleted lipoprotein. Fifty $\mu l$ of the sonicated mixture was obtained and added to the incubation mixture. The protein concentrations of apo-HDL and apo-LDL were 7.9 $\mu \mathrm{g} /$ assay and $3.2 \mu \mathrm{g}$ /assay, respectively. Postnuclear supernatant (100 $\mu \mathrm{g}$ of protein per assay) was used. Results are expressed as the mean \pm SEM. ( $n=4$ different experiments). 


\section{References}

1. Morris MD, Chaikoff IL (1959) The origin of cholesterol in liver, small intestine, adrenal gland, and testis of the rat: Dietary versus endogenous contributions. J Biol Chem 234: 1095-1097.

2. Dexter RN, Fishman LN, Ney RL (1970) Stimulation of adrenal cholesterol uptake from plasma by adrenocorticotropin. Endocrinology 87: 836-846.

3. Borkowski AJ, Levin S, Delcroix C, Klatersky J (1970) Equilibration of plasma and adrenal cholesterol in man. J Appl Physiol 28: 42-49.

4. Borkowski AJ, Delcroix C, Levin S (1972) Metabolism of adrenal cholesterol in man. 1. in vivo studies. J Clin Invest 51: 1664-1678.

5. Borkowski AJ, Delcroix C, Levin S (1972) Metabolism of adrenal cholesterol in man. 2. in vivo studies including a comparison of adrenal cholesterol synthesis with the synthesis of the glucocorticoid hormones. J Clin Invest 51: 1679-1687.

6. Faust JR, Goldstein JL, Brown MS (1977) Receptor-mediated uptake of low density lipoprotein and utilization of its cholesterol for steroid synthesis in cultured mouse adrenal cells. J Biol Chem 252: 4861-4871.

7. Hall PF, Nakamura M (1979) The influence of adrenocorticotropin on transport of a cholesteryl linoleate-low density lipoprotein complex into adrenal tumor cells. J Biol Chem 254: 12547-12554.

8. Sholl SA, Kupsky SE, Wolf RC (1981) In vivo uptake and metabolism of low density lipoprotein in the guinea pig adrenal. Steroids 37: 485-495.

9. Carr BR, Parker Jr CR, Milewich L, Porter JC, MacDonald PC, Simpson ER (1980) The role of low density, high density, and very low density lipoprotein in steroidogenesis by human fetal adrenal gland. Endocrinology 106: 1854-1860.

10. Anderson JM, Dietschy JM (1978) Relative importance of high and low density lipoproteins in the regulation of cholesterol synthesis in the adrenal gland, ovary, and testis of the rat. J Biol Chem 253: 9024-9032.

11. Gwynne JT, Hess B (1980) The role of high density lipoproteins in rat adrenal cholesterol metabolism and steroidogenesis. J Biol Chem 255: 10875-10883.

12. Gwynne JT, Mahaffee DD (1989) Rat adrenal uptake and metabolism of high density lipoprotein cholesteryl ester. J Biol Chem 264: 8141-8150.

13. Nishikawa T, Mikami K, Saito Y, Tamura Y, Kumagai A (1981) Studies on cholesterol esterase in the rat adrenal. Endocrinology 108: 932-936.

14. Brecher P, Pyun HY, Chobanian AV (1978) Cholesteryl ester and triglyceride hydrolysis by an acid lipase from rabbit aorta. Biochem Biophy Acta 530: 112-113.

15. Yamamoto K, Kamo-Yamada F, Cho S, Sugano M (1980) Some observations on the cholesterol esterifying and cholesterol ester hydrolyzing activities in dog plasma. J Biochem (Tokyo) 87: 1271-1278.

16. Gwynne JT, Mahaffee D, Brewer Jr HB, Ney RL (1976) Adrenal cholesterol uptake from plasma lipoproteins: Regulation by corticotropin. Proc Natl Acad Sci USA 73: 4329-4333.

17. Nicosia M, Prack MM, Williams DL (1992) Differential regulation of apolipoprotein-E messenger RNA in zona fasciculata cells of rat adrenal gland determined by the in situ hybridization. Mol Endocrinol 6: 288-298.

18. Lowry $\mathrm{OH}$, Rosebrough NJ, Farr AL, Randall RJ (1951) Protein measurement with Folin phenol reagent. J Biol Chem 193: 265-275.

19. Koga S, Horwitz DL, Scanu AN (1969) Isolation and properties of lipoproteins from normal rat serum. J Lipid Res 10: 577-588.

20. Nishikawa T, Mikami K, Tamura Y, Yamamoto M, Kumagai A (1979) Comparative study of cyclic AMP-generation system, steroid biosynthesis and lipid metabolism in vitro in ACTH responsive and unresponsive adrenal tumors. Endocrinol Japon 26: 9-17.

21. Scanu AN (1966) Forms of human serum high density lipoprotein protein. J Lipid Res 7: 295-306.

22. Nishikawa T, Mikami K, Saito Y, Tamura Y, Yoshida $S$ (1988) Functional differences in cholesterol ester hydrolase and acyl-coenzymeA/cholesterol acyltransferase between the outer and inner zones of the guinea pig adrenal cortex. Endocrinology 122: 877-883.

23. Boyd GS, Trezeciak WH (1973) Studies on the mode of action of ACTH. Ann NY Acad Sci 212: 361-377.

24. Mahley RW, Holcombe KS (1977) Alterations of the plasma lipoproteins and apoproteins following cholesterol feeding in the rat. $J$ Lipid Res 18: 314-324.

25. Swaney JB, Braithwaite F, Eder HA (1977) Characterization of the apolipoproteins of rat plasma lipoproteins. Biochemistry 16: 271-278. 